\title{
Existence and stability of Langevin equations with two Hilfer-Katugampola fractional derivatives
}

\author{
Rabha W. Ibrahim, Sugumaran Harikrishnan and Kuppusamy \\ Kanagarajan
}

\begin{abstract}
In this note, we debate the existence, uniqueness and stability results for a general class of Langevin equations. We suggest the generalization via the Hilfer-Katugampola fractional derivative. We introduce some conditions for existence and uniqueness of solutions. We utilize the concept of fixed point theorems (Krasnoselskii fixed point theorem (KFPT), Banach contraction principle $(\mathrm{BCP}))$. Moreover, we illustrate definitions of the Ulam type stability. These definitions generalize the fractional Ulam stability.
\end{abstract}

Mathematics Subject Classification (2010): 26A33, 49K40.

Keywords: Fractional calculus, fractional differential operator, fractional differential equation, Ulam stability.

\section{Introduction}

The field of an arbitrary calculus (fractional calculus) is the extension of the ordinary calculus of fractional powers. It plays a significant field in the mathematical analysis. In addition, it is more than three centuries old, yet it only receives much attention and interest in last three decades [7, 17, 20]. The Langevin equation describes the stochastic problem in many fluctuating situations. A modified type of this equation used in various functional approaches of fractal mediums. Another modification requires replacing of ordinary differential equations into fractional differential equations (FDE), which yields the fractional Langevin equation $[2,4,5,6,3,21]$.

In recent times, Katugampola [13] introduced a new fractional differential operator which studied extensively by many researchers [14, 15, 25, 26]. Moreover, this operator has been compounded with Hilfer fractional differential operator introduced by Hilfer [7] to develop a new fractional differential operator, so called Hilfer-Katugampola fractional differential operator [19]. For the wide knowledge of 
fractional differential operators, one can refer to [22, 23, 24]. Rassias imposed the Hyers-Ulam stability (UHS) for both cases linear and nonlinear studies. This outcome of Rassias attracted many investigators worldwide who began to be motivated to study the stability problems of differential equations $[1,12,18,29]$. The fractional Ulam stability (FUS) introduced by Wang [28], [27] and Ibrahim [8]-[11]. In our investigation, we focus on the following fractional differential equation containing two Hilfer-Katugampola fractional differential operators

$$
\left\{\begin{array}{l}
{ }^{\rho} D^{\alpha_{1}, \beta}\left({ }^{\rho} D^{\alpha_{2}, \beta}+\lambda\right) x(t)=f(t, x(t)), \quad t \in J:=(a, b] \\
I^{1-\gamma} x(a)=x_{a}, \quad \gamma=\left(\alpha_{1}+\alpha_{2}\right)(1-\beta)+\beta .
\end{array}\right.
$$

where ${ }^{\rho} D^{\alpha_{1}, \beta}$ and ${ }^{\rho} D^{\alpha_{2}, \beta}$ is Hilfer-Katugampola fractional differential operator of orders $\alpha_{1}$ and $\alpha_{2}$ and type $\beta, \rho>0$ and $\lambda$ is any real number. Let $f: J \times R \rightarrow R$ is given continuous function.

The effort is systematic as follows: In Section 2, we submit preliminaries that utilized throughout the paper. In Section 3, we set up the existence and uniqueness for a special formula of multi-power FDE covering the Hilfer-Katugampola fractional differential operator. In Section 4, we discuss some types of fractional Ulam stability.

\section{Preliminaries}

Some basic definitions and results introduced in the recent section. The following observations selected from $[17,14,19]$. Let $C[a, b]$ be a space of all continuous functions subject to the sup. norm $\|\psi\|=\sup \{|\psi(t)|: t \in J\}$. The weighted space $C_{\gamma, \rho}[a, b]$ of functions $f$ on $(a, b]$ is defined by

$$
C_{\gamma, \rho}[a, b]=\left\{f:(a, b] \rightarrow R:\left(\frac{t^{\rho}-a^{\rho}}{\rho}\right)^{\gamma} f(x) \in C[a, b]\right\}, 0 \leq \gamma<1,
$$

with the norm

$$
\|g\|_{C_{\gamma, \rho}}=\left\|\left(\frac{t^{\rho}-a^{\rho}}{\rho}\right)^{\gamma} f(x)\right\|_{C}=\max _{t \in J}\left|\left(\frac{t^{\rho}-a^{\rho}}{\rho}\right)^{\gamma} f(x)\right|, C_{0, \rho}[a, b]=C[a, b] .
$$

Let $\delta_{\rho}=\left(t^{\rho} \frac{d}{d t}\right)$ and for $n \in N$, the notion $C_{\delta_{\rho, \gamma}}^{n}[a, b]$, be the Banach space of all functions $f$ which are continuously differentiable. Suppose that the operator $\delta_{\rho}$, is on $[a, b]$ of $(n-1)$-order and the derivative $\delta_{\rho}^{n} f$ of $n$-order on $(a, b]$ such that $\delta_{\rho}^{n} f \in$ $C_{\gamma, \rho}[a, b]$. This leads to

$$
C_{\delta_{\rho, \gamma}}^{n}[a, b]=\left\{\delta_{\rho}^{k} f \in C[a, b], \delta_{\rho}^{n} f \in C_{\gamma, \rho}[a, b], k=0,1, \ldots, n-1\right\}
$$

with the norm

$$
\|f\|_{C_{\delta_{\rho, \gamma}}^{n}}=\sum_{k=0}^{n-1}\left\|\delta_{\rho}^{k} f\right\|_{C}+\left\|\delta_{\rho}^{n} f\right\|_{C_{\gamma, \rho}}, \quad\|f\|_{C_{\delta_{\rho}}^{n}}=\sum_{k=0}^{n} \max _{x \in R}\left|\delta_{\rho}^{k} f(x)\right| .
$$

For $n=0$, we have

$$
C_{\delta_{\rho, \gamma}}^{0}[a, b]=C_{\gamma, \rho}[a, b]
$$


Definition 2.1. Let $\alpha, c \in R$ with $\alpha>0$ and $f \in X_{c}^{p}(a, b)$, where $f \in X_{c}^{p}(a, b)$ consists of the Lebesgue measurable functions. The generalized left-sided fractional integral ${ }^{\rho} I_{a^{+}}^{\alpha} f$ of order $\alpha \in C(\Re(\alpha))$ is defined by

$$
\left({ }^{\rho} I_{a^{+}}^{\alpha} f\right)(t)=\frac{\rho^{1-\alpha}}{\Gamma(\alpha)} \int_{a}^{t}\left(t^{\rho}-s^{\rho}\right)^{\alpha-1} s^{\rho-1} f(s) d s, t>a .
$$

The extended fractional derivative analog to the extended fractional integral $(2.1)$, is given by

$$
\left({ }^{\rho} D_{a^{+}}^{\alpha} f\right)(t)=\frac{\rho^{\alpha-n-1}}{\Gamma(n-\alpha)}\left(t^{1-\rho} \frac{d}{d t}\right)^{n} \int_{a}^{t}\left(t^{\rho}-s^{\rho}\right)^{n-\alpha+1} s^{\rho-1} f(s) d s .
$$

Definition 2.2. The Hilfer-Katugampola fractional operator with respect to $t$, of order $\rho>0$, is defined by

$$
\begin{aligned}
\left({ }^{\rho} D_{a^{ \pm}}^{\alpha, \beta} f\right)(t) & =\left( \pm^{\rho} I_{a^{ \pm}}^{\alpha}\left(t^{\rho-1} \frac{d}{d t}\right){ }^{\rho} I_{a^{ \pm}}^{(1-\beta)(1-\alpha)}\right)(t) \\
& =\left( \pm^{\rho} I_{a^{ \pm}}^{\alpha} \delta_{\rho}{ }^{\rho} I_{a^{ \pm}}^{(1-\beta)(1-\alpha)}\right)(t) .
\end{aligned}
$$

- The operator ${ }^{\rho} D_{a^{+}}^{\alpha, \beta}$ can be written as

$$
{ }^{\rho} D_{a^{+}}^{\alpha, \beta}={ }^{\rho} I_{a^{+}}^{\beta(1-\alpha)} \delta_{\rho}{ }^{\rho} I_{a^{+}}^{1-\gamma}={ }^{\rho} I_{a^{+}}^{\beta(1-\alpha)} \rho D_{a^{+}}^{\gamma}, \quad \gamma=\alpha+\beta-\alpha \beta .
$$

- The fractional derivative ${ }^{\rho} D_{a^{+}}^{\alpha, \beta}$ is considered as interpolation, with the convenient parameters, of the following fractional derivatives, Hilfer fractional operator when $\rho \rightarrow 1$, Hilfer-Hadamard operator when $\rho \rightarrow 0$, generalized fractional operator when $\beta=0$, Caputo-type fractional derivative when $\beta=1$, RiemannLiouville fractional derivative when $\beta=0, \rho \rightarrow 1$, Hadamard operator when $\beta=0, \rho \rightarrow 0$, Caputo operator when $\beta=1, \rho \rightarrow 1$, Caputo-Hadamard operator when $\beta=1, \rho \rightarrow 0$, Liouville fractional derivative when $\beta=0, \rho \rightarrow 1, a=0$ and Hadamard fractional derivative when $\beta=0, \rho \rightarrow 1, a=-\infty$. We consider the following parameters $\alpha, \beta, \gamma, \mu$, :

$$
\gamma=\alpha+\beta-\alpha \beta, \quad 0 \leq \mu<1, \alpha>0, \beta<1,0 \leq \gamma<1 .
$$

The following results can be found in [19]:

Lemma 2.3. Let $\alpha, \beta>0,0<a<b<\infty, \rho, c \in R, 1 \leq p \leq \infty$ and $\rho \geq c$. Then, for $f \in X_{c}^{p}(a, b)$ the semi group property is valid. This is,

$$
\left({ }^{\rho} I_{a^{+}}^{\alpha}{ }^{\rho} I_{a^{+}}^{\beta} f\right)(x)=\left({ }^{\rho} I_{a^{+}}^{\alpha+\beta}\right)(x),
$$

and

$$
\left({ }^{\rho} D_{a^{+}}^{\alpha}{ }^{\rho} I_{a^{+}}^{\alpha} f\right)(x)=f(x) .
$$


Lemma 2.4. Assume that $x>a,{ }^{\rho} I_{a^{+}}^{\alpha}$ and ${ }^{\rho} D_{a^{+}}^{\alpha}$ are according on Eq. (2.1) and (2.2), respectively. Then

$$
\begin{aligned}
& { }^{\rho} I_{a^{+}}^{\alpha}\left(\frac{t^{\rho}-a^{\rho}}{\rho}\right)^{\beta-1}(x)=\left(\frac{x^{\rho}-a^{\rho}}{\rho}\right)^{\alpha+\beta-1} \frac{\Gamma(\beta)}{\Gamma(\alpha+\beta)}, \alpha \geq 0, \\
& { }^{\rho} D_{a^{+}}^{\alpha}\left(\frac{t^{\rho}-a^{\rho}}{\rho}\right)^{\beta-1}(x)=0, \quad \alpha \in(0,1), \beta \in(0, \infty) .
\end{aligned}
$$

Lemma 2.5. Let $0<\alpha<1,0 \leq \gamma<1$. If $f \in C_{\gamma}[a, b]$ and ${ }^{\rho} I_{a+}^{1-\alpha} f \in C_{\gamma}^{1}[a, b]$, then

$$
\left({ }^{\rho} I_{a^{+}}^{\alpha}{ }^{\rho} D_{a^{+}}^{\alpha} f\right)(x)=f(x)-\frac{\left({ }^{\rho} I_{a+}^{1-\alpha} f\right)(a)}{\Gamma(\alpha)}\left(\frac{x^{\rho}-a^{\rho}}{\rho}\right)^{\alpha-1},
$$

for all $x \in(a, b]$.

Lemma 2.6. Let $0<a<b<\infty, \alpha>0,0 \leq \gamma<1$ and $f \in C_{\gamma, \rho}[a, b]$. If $\alpha>\gamma$, then ${ }^{\rho} I_{a^{+}}^{\alpha} f$ is continuous on $[a, b]$ and

$$
\left({ }^{\rho} I_{a^{+}}^{\alpha} f\right)(a)=\lim _{t \rightarrow a^{+}}\left({ }^{\rho} I_{a^{+}}^{\alpha} f\right)(t)=0 .
$$

We present some spaces as follows:

$$
C_{1-\gamma, \rho}^{\alpha, \beta}[a, b]=\left\{f \in C_{1-\gamma, \rho}[a, b],{ }^{\rho} D_{a^{+}}^{\alpha, \beta} f \in C_{\mu, \rho}[a, b]\right\}
$$

and

$$
C_{1-\gamma, \rho}^{\gamma}[a, b]=\left\{f \in C_{1-\gamma, \rho}[a, b],{ }^{\rho} D_{a^{+}}^{\gamma} f \in C_{1-\gamma, \rho}[a, b]\right\} .
$$

Clearly, we have

$$
C_{1-\gamma, \rho}^{\gamma}[a, b] \subset C_{1-\gamma, \rho}^{\alpha, \beta}[a, b] .
$$

Lemma 2.7. If $C_{1-\gamma}^{\gamma}[a, b]$, then

$$
{ }^{\rho} I_{a^{+}}^{\gamma}{ }^{\rho} D_{a^{+}}^{\gamma} f={ }^{\rho} I_{a^{+}}^{\alpha}{ }^{\rho} D_{a^{+}}^{\alpha, \beta} f
$$

and

$$
{ }^{\rho} D_{a^{+}}^{\gamma}{ }^{\rho} I_{a^{+}}^{\alpha} f={ }^{\rho} D_{a^{+}}^{\beta(1-\alpha)} f .
$$

Lemma 2.8. If ${ }^{\rho} D_{a^{+}}^{\beta(1-\alpha)} f$ exists on $L^{1}(a, b)$, then

$$
{ }^{\rho} D_{a^{+}}^{\alpha, \beta \rho} I_{a^{+}}^{\alpha} f={ }^{\rho} I_{a^{+}}^{\beta(1-\alpha) \rho} D_{a^{+}}^{\beta(1-\alpha)} f .
$$

Lemma 2.9. Let $0<\alpha<1,0 \leq \beta \leq 1$ and $\gamma=\alpha+\beta-\alpha \beta$. If $f \in C_{1-\gamma}[a, b]$ and ${ }^{\rho} I_{a^{+}}^{1-\beta(1-\alpha)} \in C_{1-\gamma}^{1-}[a, b]$, then ${ }^{\rho} D_{a^{+}}^{\alpha, \beta} I_{a^{+}}^{\alpha}$ exists on $(a, b]$ and

$$
{ }^{\rho} D_{a^{+}}^{\alpha, \beta} I_{a^{+}}^{\alpha} f=f
$$

Lemma 2.10. [19] Let $\gamma=\alpha+\beta-\alpha \beta$. If $f:(a, b] \times R \rightarrow R$ is a function such that $f(\cdot, x(\cdot)) \in C_{1-\gamma, \rho}[a, b]$ for all $x \in C_{1-\gamma, \rho}[a, b]$ then a function $x \in C_{1-\gamma, \rho}^{\gamma}[a, b]$ is the outcome of the problem

$$
\left\{\begin{array}{l}
{ }^{\rho} D_{a^{+}}^{\alpha_{1}, \beta}\left({ }^{\rho} D_{a^{+}}^{\alpha_{2}, \beta}+\lambda\right) x(t)=f(t, x(t)), t \in(a, b], \\
{ }^{\rho} I_{a_{+}}^{1-\gamma} x(a)=x_{a}
\end{array}\right.
$$


if and only if $x$ achieves the following formula:

$$
\begin{aligned}
x(t)= & \frac{x_{a}}{\Gamma(\gamma)}\left(\frac{t^{\rho}-a^{\rho}}{\rho}\right)^{\gamma-1}-\frac{\lambda}{\Gamma\left(\alpha_{2}\right)} \int_{a}^{t}\left(\frac{t^{\rho}-s^{\rho}}{\rho}\right)^{\alpha_{2}-1} s^{\rho-1} x(s) d s \\
& +\frac{1}{\Gamma\left(\alpha_{1}+\alpha_{2}\right)} \int_{a}^{t}\left(\frac{t^{\rho}-s^{\rho}}{\rho}\right)^{\alpha_{1}+\alpha_{2}-1} s^{\rho-1} f(s, x(s)) d s .
\end{aligned}
$$

The proof of the lemma is similar ([30], Lemma 3.1).

Theorem 2.11. (KFPT) Suppose that $\Sigma$ is a Banach space, $\Theta$ is a closed, bounded and convex subset of $\Sigma$ and two functions $\Gamma_{1}, \Gamma_{2}: \Theta \rightarrow \Sigma$ such that $\Gamma_{1} \chi+\Gamma_{2} \eta \in \Theta$ for all $\chi, \eta \in \Theta$. If $\Gamma_{1}$ is a contraction function and $\Gamma_{2}$ is completely continuous, then $\Gamma_{1} \chi+\Gamma_{2} \chi=\chi$ admits a solution in $\Theta$.

Theorem 2.12. (Arzela-Ascoli theorem) [16] A subset $F$ of $C(X)$ is relatively compact if and only if it is closed, bounded and equicontinuous.

\section{Existence and uniqueness results}

For our setting, we deliver the following assumptions:

(H1) Let $f(\cdot, x(\cdot)) \in C_{1-\gamma, \rho}^{\beta(1-\alpha)}[a, b]$ for any $x \in C_{1-\gamma, \rho}[a, b]$. There exists a positive constant $\ell$ such that

$$
|f(t, \chi)-f(t, \eta)| \leq \ell|\chi-\eta|, \quad \text { for all } \chi, \eta \in R .
$$

(H2) The constant

$$
\Omega=\left(\frac{\ell B\left(\gamma, \alpha_{1}+\alpha_{2}\right)}{\Gamma\left(\alpha_{1}+\alpha_{2}\right)}\left(\frac{b^{\rho}-a^{\rho}}{\rho}\right)^{\alpha_{1}+\alpha_{2}}+\frac{\lambda B\left(\gamma, \alpha_{2}\right)}{\Gamma\left(\alpha_{2}\right)}\left(\frac{b^{\rho}-a^{\rho}}{\rho}\right)^{\alpha_{2}}\right)<1 .
$$

(H3) There exist a nondecreasing function $\varphi: J \rightarrow R^{+}$and $\lambda_{\varphi}>0$ such that for $t \in J$

$$
{ }^{\rho} I_{a_{+}}^{\alpha_{1}+\alpha_{2}} \varphi(t) \leq \lambda_{\varphi} \varphi(t) .
$$

By applying Theorem 2.11, we have the following result:

Theorem 3.1. (Existence) Suppose that [H1] and [H2] are achieved. Then, Eq. (1.1) admits at least one outcome in $C_{1-\gamma, \rho}^{\gamma}[a, b] \subset C_{1-\gamma, \rho}^{\alpha, \beta}[a, b]$.

Proof. Define the operator $N: C_{1-\gamma, \rho}[a, b] \rightarrow C_{1-\gamma, \rho}[a, b]$, it is well defined and given by

$$
(N x)(t)=\left\{\begin{array}{l}
\frac{x_{a}}{\Gamma(\gamma)}\left(\frac{t^{\rho}-a^{\rho}}{\rho}\right)^{\gamma-1}-\frac{\lambda}{\Gamma\left(\alpha_{2}\right)} \int_{a}^{t}\left(\frac{t^{\rho}-s^{\rho}}{\rho}\right)^{\alpha_{2}-1} s^{\rho-1} x(s) d s \\
+\frac{1}{\Gamma\left(\alpha_{1}+\alpha_{2}\right)} \int_{a}^{t}\left(\frac{t^{\rho}-s^{\rho}}{\rho}\right)^{\alpha_{1}+\alpha_{2}-1} s^{\rho-1} f(s, x(s)) d s
\end{array}\right.
$$

Set $\tilde{f}(s)=f(s, 0)$ and

$$
\omega=\left(\frac{b^{\rho}-a^{\rho}}{\rho}\right)^{\alpha_{1}+\alpha_{2}} \frac{B\left(\gamma, \alpha_{1}+\alpha_{2}\right)}{\Gamma\left(\alpha_{1}+\alpha_{2}\right)}\|f\|_{C_{1-\gamma, \rho}}+\frac{x_{a}}{\Gamma(\gamma)}
$$


Consider the ball $B_{r}=\left\{\chi \in C_{1-\gamma, \rho}[a, b]:\|\chi\|_{C_{1-\gamma, \rho}} \leq r\right\}$.

Now we subdivide the operator $N$ into two operator $A$ and $B$ on $B_{r}$ as follows:

$$
(A x)(t)=\frac{1}{\Gamma\left(\alpha_{1}+\alpha_{2}\right)} \int_{a}^{t}\left(\frac{t^{\rho}-s^{\rho}}{\rho}\right)^{\alpha_{1}+\alpha_{2}-1} s^{\rho-1} f(s, x(s)) d s
$$

and

$$
(B x)(t)=\frac{x_{a}}{\Gamma(\gamma)}\left(\frac{t^{\rho}-a^{\rho}}{\rho}\right)^{\gamma-1}-\frac{\lambda}{\Gamma\left(\alpha_{2}\right)} \int_{a}^{t}\left(\frac{t^{\rho}-s^{\rho}}{\rho}\right)^{\alpha_{2}-1} s^{\rho-1} x(s) d s .
$$

The proof is as follows:

Step 1. $A x+B y \in B_{r}$ for every $x, y \in B_{r}$.

$$
\begin{aligned}
& \left|(A x)(t)\left(\frac{t^{\rho}-a^{\rho}}{\rho}\right)^{1-\gamma}\right| \\
& \leq\left(\frac{t^{\rho}-a^{\rho}}{\rho}\right)^{1-\gamma} \frac{1}{\Gamma\left(\alpha_{1}+\alpha_{2}\right)} \int_{a}^{t}\left(\frac{t^{\rho}-s^{\rho}}{\rho}\right)^{\alpha_{1}+\alpha_{2}-1} s^{\rho-1} \times f(s, x(s)) d s \\
& \leq\left(\frac{t^{\rho}-a^{\rho}}{\rho}\right)^{1-\gamma} \frac{1}{\Gamma\left(\alpha_{1}+\alpha_{2}\right)} \int_{a}^{t}\left(\frac{t^{\rho}-s^{\rho}}{\rho}\right)^{\alpha_{1}+\alpha_{2}-1} s^{\rho-1}(|f(s, x(s))-f(s, 0)|+|f(s, 0)|) d s \\
& \leq\left(\frac{t^{\rho}-a^{\rho}}{\rho}\right)^{1-\gamma} \frac{1}{\Gamma\left(\alpha_{1}+\alpha_{2}\right)} \int_{a}^{t}\left(\frac{t^{\rho}-s^{\rho}}{\rho}\right)^{\alpha_{1}+\alpha_{2}-1} s^{\rho-1}(\ell|x(s)|+|\tilde{f}(s)|) d s \\
& \leq\left(\frac{t^{\rho}-a^{\rho}}{\rho}\right)^{1-\gamma} \frac{B\left(\gamma, \alpha_{1}+\alpha_{2}\right)}{\Gamma\left(\alpha_{1}+\alpha_{2}\right)}\left(\frac{t^{\rho}-a^{\rho}}{\rho}\right)^{\alpha_{1}+\alpha_{2}+\gamma-1}\left(\ell\|x\|_{C_{1-\gamma}}+\|\tilde{f}\|_{C_{1-\gamma, \rho}}\right) .
\end{aligned}
$$

This gives

$$
\|A x\|_{C_{1-\gamma, \rho}} \leq \frac{B\left(\gamma, \alpha_{1}+\alpha_{2}\right)}{\Gamma\left(\alpha_{1}+\alpha_{2}\right)}\left(\frac{b^{\rho}-a^{\rho}}{\rho}\right)^{\alpha_{1}+\alpha_{2}}\left(\ell\|x\|_{C_{1-\gamma, \rho}}+\|\tilde{f}\|_{C_{1-\gamma, \rho}}\right) .
$$

For operator $B$

$$
\begin{aligned}
& \left|(B x)(t)\left(\frac{t^{\rho}-a^{\rho}}{\rho}\right)^{1-\gamma}\right| \\
& \leq \frac{x_{a}}{\Gamma(\gamma)}+\left(\frac{t^{\rho}-a^{\rho}}{\rho}\right)^{1-\gamma} \frac{\lambda}{\Gamma\left(\alpha_{2}\right)} \int_{a}^{t}\left(\frac{t^{\rho}-s^{\rho}}{\rho}\right)^{\alpha_{2}-1} s^{\rho-1} x(s) d s \\
& \leq \frac{x_{a}}{\Gamma(\gamma)}+\left(\frac{t^{\rho}-a^{\rho}}{\rho}\right)^{1-\gamma} \frac{\lambda B\left(\gamma, \alpha_{2}\right)}{\Gamma\left(\alpha_{2}\right)}\left(\frac{t^{\rho}-a^{\rho}}{\rho}\right)^{\alpha_{2}+\gamma-1}\|x\|_{C_{1-\gamma, \rho}} .
\end{aligned}
$$

Thus, we obtain

$$
\|(B x)\|_{C_{1-\gamma}} \leq \frac{x_{a}}{\Gamma(\gamma)}+\frac{\lambda B\left(\gamma, \alpha_{2}\right)}{\Gamma\left(\alpha_{2}\right)}\left(\frac{b^{\rho}-a^{\rho}}{\rho}\right)^{\alpha_{2}}\|x\|_{C_{1-\gamma, \rho}} .
$$

Linking (3.2) and (3.3), for every $x, y \in B_{r}$, we get

$$
\|A x+B y\|_{C_{1-\gamma, \rho}} \leq\|A x\|_{C_{1-\gamma, \rho}}+\|B y\|_{C_{1-\gamma, \rho}} \leq \Omega r+\omega .
$$


Step 2. $A$ is a contraction mapping.

For any $x, y \in B_{r}$, we observe the conclusion

$$
\begin{aligned}
& \left|((A x)(t)-(A y)(t))\left(\frac{t^{\rho}-a^{\rho}}{\rho}\right)^{1-\gamma}\right| \\
& \leq\left(\frac{t^{\rho}-a^{\rho}}{\rho}\right)^{1-\gamma} \frac{1}{\Gamma\left(\alpha_{1}+\alpha_{2}\right)} \int_{a}^{t}\left(\frac{t^{\rho}-s^{\rho}}{\rho}\right)^{\alpha_{1}+\alpha_{2}-1} s^{\rho-1}|f(s, x(s))-f(s, y(s))| d s \\
& \leq\left(\frac{t^{\rho}-a^{\rho}}{\rho}\right)^{1-\gamma} \frac{\ell}{\Gamma\left(\alpha_{1}+\alpha_{2}\right)}\left(\frac{t^{\rho}-a^{\rho}}{\rho}\right)^{\alpha_{1}+\alpha_{2}+\gamma-1}\|x-y\|_{C_{1-\gamma, \rho}} .
\end{aligned}
$$

This gives

$$
\|(A x)-(A y)\| \leq \frac{\ell B\left(\gamma, \alpha_{1}+\alpha_{2}\right)}{\Gamma\left(\alpha_{1}+\alpha_{2}\right)}\left(\frac{b^{\rho}-a^{\rho}}{\rho}\right)^{\alpha_{1}+\alpha_{2}}\|x-y\|_{C_{1-\gamma, \rho}} .
$$

In view of [H2], the operator $A$ is a contraction mapping.

Step 3. The operator $B$ is completely compact.

According to Step 1, we know that

$$
\|(B x)\|_{C_{1-\gamma, \rho}} \leq \frac{x_{a}}{\Gamma(\gamma)}+\frac{\lambda B\left(\gamma, \alpha_{2}\right)}{\Gamma\left(\alpha_{2}\right)}\left(\frac{b^{\rho}-a^{\rho}}{\rho}\right)^{\alpha_{2}}\|x\|_{C_{1-\gamma, \rho}} .
$$

Thus, the operator $B$ is uniformly bounded. Next, we show that the operator $B$ is compact. A calculation implies

$$
\begin{aligned}
\left|(B x)\left(t_{1}\right)-(B x)\left(t_{2}\right)\right| & \leq \frac{x_{a}}{\Gamma(\gamma)}\left|\left(\frac{t_{1}^{\rho}-a^{\rho}}{\rho}\right)^{\gamma-1}-\left(\frac{t_{2}^{\rho}-a^{\rho}}{\rho}\right)^{\gamma-1}\right| \\
& +\frac{\ell B\left(\gamma, \alpha_{2}\right)}{\Gamma\left(\alpha_{2}\right)}\|x\|_{C_{1-\gamma, \rho}}\left|\left(\frac{t_{1}^{\rho}-a^{\rho}}{\rho}\right)^{\alpha_{2}+\gamma-1}-\left(\frac{t_{2}^{\rho}-a^{\rho}}{\rho}\right)^{\alpha_{2}+\gamma-1}\right|
\end{aligned}
$$

which is tending to zero as $t_{1} \rightarrow t_{2}$. Thus $B$ is equicontinuous. Hence, in virtue of the Theorem 2.12, the operator $B$ is compact on $B_{r}$. It leads by Krasnoselskii fixed point theorem, that the problem (1.1) admits a solution.

Theorem 3.2. If hypothesis (H1) and (H2) are fulfilled. Then, Eq. (1.1) admits a unique solution.

\section{Stability outcomes}

In the recent section, we shall give the definitions and the criteria of (UHS) and (UHRS) for the generalized Langevin Eq. (1.1). Now for $\epsilon>0$ and a continuous function $\varphi: J \rightarrow R^{+}$, we theorize the next inequalities:

$$
\begin{gathered}
\left|{ }^{\rho} D_{a^{+}}^{\alpha_{1}, \beta}\left({ }^{\rho} D_{a^{+}}^{\alpha_{2}, \beta}+\lambda\right) z(t)-f(t, z(t))\right| \leq \epsilon, \quad t \in J, \\
\left|{ }^{\rho} D_{a^{+}}^{\alpha_{1}, \beta}\left({ }^{\rho} D_{a^{+}}^{\alpha_{2}, \beta}+\lambda\right) z(t)-f(t, z(t))\right| \leq \epsilon \varphi(t), \quad t \in J,
\end{gathered}
$$




$$
\left|{ }^{\rho} D_{a^{+}}^{\alpha_{1}, \beta}\left({ }^{\rho} D_{a^{+}}^{\alpha_{2}, \beta}+\lambda\right) z(t)-f(t, z(t))\right| \leq \varphi(t), \quad t \in J
$$

\section{Qualifier}

The Eq. (1.1) is UHS if there occurs a number $C_{f}>0$ and $\epsilon>0$ such that for all outcome $z \in C_{1-\gamma, \rho}[a, b]$ of the inequality (4.1) there occurs an outcome $x \in$ $C_{1-\gamma, \rho}[a, b]$ of Eq. (1.1) satisfying

$$
|z(t)-x(t)| \leq C_{f} \epsilon, \quad t \in J .
$$

The Eq. (1.1) is generalized UHS if there occurs a function $\varphi \in C_{1-\gamma, \rho}[a, b]$, $\varphi_{f}(0)=0$ such that for all outcome $z \in C_{1-\gamma, \rho}[a, b]$ of the inequality (4.1) there occurs an outcome $x \in C_{1-\gamma, \rho}[a, b]$ of Eq. (1.1) achieving

$$
|z(t)-x(t)| \leq \varphi_{f} \epsilon, \quad t \in J
$$

The Eq. (1.1) is UHRS esteeming by $\varphi \in C_{1-\gamma, \rho}[a, b]$ if there occurs a number $C_{f, \varphi}>0$ for all $\epsilon>0$ and for every outcome $z \in C_{1-\gamma, \rho}[a, b]$ of the inequality (4.2) there occurs an outcome $x \in C_{1-\gamma, \rho}[a, b]$ of Eq. (1.1) filing

$$
|z(t)-x(t)| \leq C_{f, \varphi} \epsilon \varphi(t), \quad t \in J .
$$

The Eq. (1.1) is generalized UHRS corresponding to $\varphi \in C_{1-\gamma, \rho}[a, b]$ if there occurs a real number $C_{f, \varphi}>0$ whenever for every outcome $z \in C_{1-\gamma, \rho}[a, b]$ of the inequality (4.3) there occurs an outcome $x \in C_{1-\gamma, \rho}[a, b]$ of Eq. (1.1) satisfying

$$
|z(t)-x(t)| \leq C_{f, \varphi} \varphi(t), \quad t \in J
$$

Remark 4.1. A function $z \in C_{1-\gamma, \rho}[a, b]$ is an outcome of the inequality (4.1) if and only if there exists a function $g \in C_{1-\gamma, \rho}[a, b]$ such that

$$
\left|\rho D_{a^{+}}^{\alpha_{1}, \beta}\left({ }^{\rho} D_{a^{+}}^{\alpha_{2}, \beta}+\lambda\right) z(t)-f(t, z(t))\right| \leq \epsilon, \quad t \in J,
$$

if and only if there occurs a function $g \in C_{1-\gamma, \rho}[a, b]$ such that

(i) $|g(t)| \leq \epsilon, t \in J$.

(ii) ${ }^{\rho} D_{a^{+}}^{\alpha_{1}, \beta}\left({ }^{\rho} D_{a^{+}}^{\alpha_{2}, \beta}+\lambda\right) z(t)=f(t, z(t))+g(t), t \in J$.

Similarly, for the inequalities (4.2) and (4.3).

Remark 4.2. If $z$ is an outcome of (4.1), then $z$ is an outcome of the following formula:

$$
\begin{aligned}
& \mid z(t)-\frac{z_{a}}{\Gamma(\gamma)}\left(\frac{t^{\rho}-a^{\rho}}{\rho}\right)^{\gamma-1}+\frac{\lambda}{\Gamma\left(\alpha_{2}\right)} \int_{a}^{t}\left(\frac{t^{\rho}-s^{\rho}}{\rho}\right)^{\alpha_{2}-1} s^{\rho-1} z(s) d s \\
& -\frac{1}{\Gamma\left(\alpha_{1}+\alpha_{2}\right)} \int_{a}^{t}\left(\frac{t^{\rho}-s^{\rho}}{\rho}\right)^{\alpha_{1}+\alpha_{2}-1} s^{\rho-1} f(s, z(s)) d s \mid \\
& \leq \frac{1}{\Gamma\left(\alpha_{1}+\alpha_{2}+1\right)}\left(\frac{b^{\rho}-a^{\rho}}{\rho}\right)^{\alpha_{1}+\alpha_{2}} \epsilon .
\end{aligned}
$$

It is clear that

$$
{ }^{\rho} D_{a^{+}}^{\alpha_{1}, \beta}\left({ }^{\rho} D_{a^{+}}^{\alpha_{2}, \beta}+\lambda\right) z(t)=f(t, z(t))+g(t), t \in J .
$$


Then

$$
\begin{aligned}
z(t) & =\frac{z_{a}}{\Gamma(\gamma)}\left(\frac{t^{\rho}-a^{\rho}}{\rho}\right)^{\gamma-1}-\frac{\lambda}{\Gamma\left(\alpha_{2}\right)} \int_{a}^{t}\left(\frac{t^{\rho}-s^{\rho}}{\rho}\right)^{\alpha_{2}-1} s^{\rho-1} z(s) d s \\
& +\frac{1}{\Gamma\left(\alpha_{1}+\alpha_{2}\right)} \int_{a}^{t}\left(\frac{t^{\rho}-s^{\rho}}{\rho}\right)^{\alpha_{1}+\alpha_{2}-1} s^{\rho-1}(f(s, z(s))+g(s)) d s
\end{aligned}
$$

Consequently, we obtain

$$
\begin{aligned}
& \mid z(t)-\frac{z_{a}}{\Gamma(\gamma)}\left(\frac{t^{\rho}-a^{\rho}}{\rho}\right)^{\gamma-1}+\frac{\lambda}{\Gamma\left(\alpha_{2}\right)} \int_{a}^{t}\left(\frac{t^{\rho}-s^{\rho}}{\rho}\right)^{\alpha_{2}-1} s^{\rho-1} z(s) d s \\
& -\frac{1}{\Gamma\left(\alpha_{1}+\alpha_{2}\right)} \int_{a}^{t}\left(\frac{t^{\rho}-s^{\rho}}{\rho}\right)^{\alpha_{1}+\alpha_{2}-1} s^{\rho-1} f(s, z(s)) d s \mid \\
& \leq \frac{1}{\Gamma\left(\alpha_{1}+\alpha_{2}\right)} \int_{a}^{t}\left(\frac{t^{\rho}-s^{\rho}}{\rho}\right)^{\alpha_{1}+\alpha_{2}-1} s^{\rho-1}|g(s)| d s \\
& \leq \frac{1}{\Gamma\left(\alpha_{1}+\alpha_{2}+1\right)}\left(\frac{b^{\rho}-a^{\rho}}{\rho}\right)^{\alpha_{1}+\alpha_{2}} \epsilon .
\end{aligned}
$$

We have similar remarks for the inequality (4.2) and (4.3).

Our main result is as follows:

Theorem 4.3. Suppose that the hypotheses [H1] and [H3] achieved. Then Eq. (1.1) is a generalized UHRS.

Proof. Let $z$ be a solution of 4.3. In view of Theorem 3.2, there $x$ is a unique outcome of the problem satisfying

$$
\begin{aligned}
{ }^{\rho} D_{a^{+}}^{\alpha_{1}, \beta}\left({ }^{\rho} D_{a^{+}}^{\alpha_{2}, \beta}+\lambda\right) x(t) & =f(t, x(t)), \\
I_{a^{+}}^{1-\gamma} x(a) & =I_{a^{+}}^{1-\gamma} z(a) .
\end{aligned}
$$

Then we have

$$
\begin{aligned}
x(t) & =\frac{z_{a}}{\Gamma(\gamma)}\left(\frac{t^{\rho}-a^{\rho}}{\rho}\right)^{\gamma-1}-\frac{\lambda}{\Gamma\left(\alpha_{2}\right)} \int_{a}^{t}\left(\frac{t^{\rho}-s^{\rho}}{\rho}\right)^{\alpha_{2}-1} s^{\rho-1} x(s) d s \\
& +\frac{1}{\Gamma\left(\alpha_{1}+\alpha_{2}\right)} \int_{a}^{t}\left(\frac{t^{\rho}-s^{\rho}}{\rho}\right)^{\alpha_{1}+\alpha_{2}-1} s^{\rho-1} f(s, x(s)) d s .
\end{aligned}
$$

By differentiating inequality (4.3), we have

$$
\begin{aligned}
\mid z(t)- & \frac{z_{a}}{\Gamma(\gamma)}\left(\frac{t^{\rho}-a^{\rho}}{\rho}\right)^{\gamma-1}+\frac{\lambda}{\Gamma\left(\alpha_{2}\right)} \int_{a}^{t}\left(\frac{t^{\rho}-s^{\rho}}{\rho}\right)^{\alpha_{2}-1} s^{\rho-1} z(s) d s \\
& -\frac{1}{\Gamma\left(\alpha_{1}+\alpha_{2}\right)} \int_{a}^{t}\left(\frac{t^{\rho}-s^{\rho}}{\rho}\right)^{\alpha_{1}+\alpha_{2}-1} s^{\rho-1} f(s, z(s)) d s \mid
\end{aligned}
$$




$$
\leq\left|\frac{1}{\Gamma\left(\alpha_{1}+\alpha_{2}\right)} \int_{0}^{t}\left(\frac{t^{\rho}-s^{\rho}}{\rho}\right)^{\alpha_{1}+\alpha_{2}-1} s^{\rho-1} \varphi(s) d s\right| \leq \lambda_{\varphi} \varphi(t) .
$$

Hence it follows that,

$$
\begin{aligned}
& |z(t)-x(t)| \\
& \leq \mid z(t)-\frac{z_{a}}{\Gamma(\gamma)}\left(\frac{t^{\rho}-a^{\rho}}{\rho}\right)^{\gamma-1}+\frac{\lambda}{\Gamma\left(\alpha_{2}\right)} \int_{a}^{t}\left(\frac{t^{\rho}-s^{\rho}}{\rho}\right)^{\alpha_{2}-1} s^{\rho-1} x(s) d s \\
& -\frac{1}{\Gamma\left(\alpha_{1}+\alpha_{2}\right)} \int_{a}^{t}\left(\frac{t^{\rho}-s^{\rho}}{\rho}\right)^{\alpha_{1}+\alpha_{2}-1} s^{\rho-1} f(s, x(s)) d s \mid \\
& \leq \mid z(t)-\frac{z_{a}}{\Gamma(\gamma)}\left(\frac{t^{\rho}-a^{\rho}}{\rho}\right)^{\gamma-1}+\frac{\lambda}{\Gamma\left(\alpha_{2}\right)} \int_{a}^{t}\left(\frac{t^{\rho}-s^{\rho}}{\rho}\right)^{\alpha_{2}-1} s^{\rho-1} z(s) d s \\
& -\frac{1}{\Gamma\left(\alpha_{1}+\alpha_{2}\right)} \int_{a}^{t}\left(\frac{t^{\rho}-s^{\rho}}{\rho}\right)^{\alpha_{1}+\alpha_{2}-1} s^{\rho-1} f(s, z(s)) d s \mid \\
& +\frac{\lambda}{\Gamma\left(\alpha_{2}\right)} \int_{a}^{t}\left(\frac{\left.t^{\rho}-s^{\rho}\right)^{\alpha_{2}-1}}{\rho}\right)^{\rho-1}|x(s)-z(s)| d s \\
& +\frac{1}{\Gamma\left(\alpha_{1}+\alpha_{2}\right)} \int_{a}^{t}\left(\frac{t^{\rho}-s^{\rho}}{\rho}\right)^{\alpha_{1}+\alpha_{2}-1} s^{\rho-1} \mid f(s, z(s))-f(s, x(s)) \| d s \\
& \leq \lambda_{\varphi} \varphi(t)+\left(\frac{\lambda}{\Gamma\left(\alpha_{2}+1\right)}\left(\frac{b^{\rho}-a^{\rho}}{\rho}\right)^{\alpha_{2}}+\frac{b^{\rho}-a^{\rho}}{\Gamma\left(\alpha_{1}+\alpha_{2}+1\right)}\right)^{\alpha_{1}+\alpha_{2}} \\
& \rho
\end{aligned}
$$

By Lemma 2.5, there occurs a constant $M^{*}>0$ independent of $\lambda_{\varphi} \varphi(t)$, achieving

$$
|z(t)-x(t)| \leq M^{*} \varphi(t)
$$

Thus, Eq. (1.1) is generalized UHRS.

Acknowledgment. The authors declare that they have no conflict of interest.

\section{References}

[1] Abbas, M.I., Ulam stability of fractional impulsive differential equations with RiemannLiouville integral boundary conditions, J. Contemp. Math. Anal., 50(2015), 209-219.

[2] Ahmad, B., Nieto, J.J., Alsaedi, A., El-Shahed, M., A study of nonlinear Langevin equation involving two fractional orders in different intervals, Nonlinear Anal., 13(2012), 599-606.

[3] Baghani, O., On fractional Langevin equation involving two fractional orders, Commun. Nonlinear. Sci. Numer. Simulat., doi:10.1016/j.cnsns.2016.05.023.

[4] Chen, A., Chen, Y., Existence of solutions to nonlinear Langevin equation involving two fractional orders with boundary value conditions, Bound. Value Probl., doi:10.1155/2011/516481.

[5] Fa, K.S., Generalized Langevin equation with fractional derivative and long-time correlation function, Phys. Rev. E, 73(2006). 
[6] Fa, K.S., Fractional Langevin equation and Riemann-Liouville fractional derivative, Eur. Phys. J. E, 24(2007), 139-143.

[7] Hilfer, R., Applications of Fractional Calculus in Physics, World scientific, Singapore, 1999.

[8] Ibrahim, R.W., Ulam-Hyers stability for Cauchy fractional differential equation in the unit disk, Abstr. Appl. Anal., 1(2012).

[9] Ibrahim, R.W., Generalized Ulam-Hyers stability for fractional differential equations, Int. J. Math., 1(2012).

[10] Ibrahim, R.W., Ulam stability for fractional differential equation in complex domain, Abstr. Appl. Anal., (2012).

[11] Ibrahim, R.W., Stability of sequential fractional differential equation, Appl. Comput. Math., (2015), 141.

[12] Kassim, M.D., Tatar, N.E., Well-posedness and stability for a differential problem with Hilfer-Hadamard fractional derivative, Abstr. Appl. Anal., (2014), 1-7.

[13] Katugampola, U.N., New approach to a genaralized fractional integral, Appl. Math. Comput., 218(2011), no. 3, 860-865.

[14] Katugampola, U.N., Existence and uniqueness results for a class of generalized fractional differential equations, Bull. Math. Anal. Appl., 1(2014).

[15] Katugampola, U.N., New fractional integral unifying six existing fractional integrals, epint arxiv: 1612.08596, 6 pages.

[16] Kelley, J.L., General Topology, Springer-Verlag, 1991.

[17] Kilbas, A.A., Srivastava, H.M., Trujillo, J.J., Theory and Applications of fractional Differential Equations, Amsterdam, Elsevier, 2006.

[18] Li, T., Zada, A., Faisal, S., Hyers-Ulam stability of nth order linear differential equations, J. Nonlinear Sci. Appl., 9(2016), 2070-2075.

[19] Oliveira, D.S., Capelas de Oliveira, E., Hilfer-Katugampola fractional derivative, arxiv:1705.07733v1, 2017.

[20] Podlubny, I., Fractional Differential Equations, Academic Press, San Diego, 1999.

[21] Torres, C., Existence of solution for fractional Langevin equation: variational approach, Electron. J. Qual. Theory Differ. Equ., 54(2014), 1-14.

[22] Vanterler da, J., Sousa, C., Capelas de Oliveira, E., On the $\psi$-Hilfer fractional derivative, arXiv: 1708.05109, 2017.

[23] Vanterler da, J., Sousa, C., Capelas de Oliveira, E., On two new operators in fractional calculus and applications, arXiv: 1710.03712, 2017.

[24] Vanterler da, J., Sousa, C., Capelas de Oliveira, E., On the Ulam-Hyers-Rassias satibility for nonlinear fractional differential equations using the $\psi$-Hilfer operator, arXiv: 1711.07339, 2017.

[25] Vivek, D., Kanagarajan, K., Harikrishnan, S., Existence and uniqueness results for pantograph equations with generalized fractional derivative, J. Nonlinear Anal. Appl., 2017.

[26] Vivek, D., Kanagarajan, K., Harikrishnan, S., Existence results for implicit differential equations with generalized fractional derivative, J. Nonlinear Anal. Appl., 2017.

[27] Wang, J., Li, X., Ulam-Hyers stability of fractional Langevin equations, Appl. Math. Comput., 258(2015), 72.

[28] Wang, J., Lv, L., Zhou, Y., Ulam stability and data dependence for fractional differential equations with Caputo derivative, Electron. J. Qual. Theory Differ. Equ., 63(2011), 1-10. 
[29] Wang, J., Zhang, Y., Ulam-Hyers-Mittag-Leffler stability of fractional-order delay differential equations, Optimization, 63(2014), 1181-1190.

[30] Yu, T., Deng, K., Luo, M., Existence and uniqueness of solutions of initial value problems for nonlinear Langevin equation involving two fractional orders, Commun. Nonlinear. Sci. Numer. Simulat., 19(2014), 1661-1668.

Rabha W. Ibrahim

Corresponding author

Faculty of Computer Science and Information Technology

University of Malaya, Kuala Lumpur 50603, Malaysia

e-mail: rabhaibrahim@yahoo.com

Sugumaran Harikrishnan

Department of Mathematics

Sri Ramakrishna Mission Vidyalaya College of Arts and Science

Coimbatore-641020, India

Kuppusamy Kanagarajan

Department of Mathematics

Sri Ramakrishna Mission Vidyalaya College of Arts and Science

Coimbatore-641020, India 